\title{
Respiratory and Baroreceptor Reflex Interactions in Man
}

\author{
Dwain L. Eckberg and Cassie R. Orshan \\ From the Cardiovascular Center, the Cardiovascular Division, the Department of Internal Medicine, \\ and the Veterans Administration and University Hospitals, University of Iowa College of Medicine, \\ Iowa City, Iowa 52242
}

\begin{abstract}
A B S T RACT Respiratory and arterial baroreceptor reflex interactions were studied in six healthy young adults. Carotid baroreceptors were stimulated with two intensities of neck suction during early inspiration or expiration at 100 or $150 \%$ of each subject's normal tidal volume. Sinus node responses to moderate baroreflex stimuli were inhibited by inspiration, but responses to intense stimuli were not influenced by the phase of respiration. Supranormal tidal volume did not diminish responses to inspiratory baroreflex stimuli, but significantly reduced responses to expiratory stimuli. These results provide evidence for a central respiratorybaroreceptor reflex interaction in man whose quality is dependent upon the level of afferent baroreceptor activity and the depth of inspiration.
\end{abstract}

\section{INTRODUCTION}

Efferent vagal cardiac activity declines or disappears altogether during inspiration $(1,2)$; and electrical carotid sinus nerve stimuli (2) or arterial pressure elevations (3) are less effective in provoking increments of efferent vagal activity or reductions of heart rate (4-6) when delivered during inspiration than expiration. These experiments suggest that neural activity associated with inspiration suppresses efferent vagal cardiac activity within the central nervous system.

The important interrelationship between respiration and baroreceptor reflexes has been studied almost exclusively in anesthetized experimental animals; there have been several obstacles preventing its definitive study in man. One series of experiments has been conducted with human volunteers, however. Smyth et al. (7) found that when arterial baroreceptors were stimulated with bolus intravenous injections of pressor drugs, pulse interval prolongation per unit sys-

Dr. Eckberg is a Clinical Investigator of the Veterans Administration. Ms. Orshan is a secondary student trainee of the National Science Foundation.

Received for publication 7 September 1976 and in revised form 21 December 1976. tolic pressure rise was slightly greater when arterial pulses occurring during inspiration were excluded from analysis. Respiratory-baroreceptor interactions have not been explored more extensively in man because, although the arterial pulse, respiration, and pulse interval (which appears to be linearly related to efferent vagal cardiac activity [3]) can be measured with precision, there has been no technique available which permits stimulation of baroreceptors during only one phase of the respiratory cycle.

We recently developed an experimental approach for stimulating human carotid baroreceptors with very brief, precisely controlled neck suction $(8,9)$. This method was used in the present study to explore central modulation of sinus node responses to baroreceptor stimulation caused by respiration. Our results provide evidence for the existence of an interaction between respiratory and baroreceptor reflexes in man, and suggest that the quality of central autonomic modulation depends upon the level of afferent baroreceptor activity and the depth of inspiration.

\section{METHODS}

Two intensities of neck suction were delivered to young adult volunteers during early inspiration or expiration, at normal or supranormal tidal volumes, and pulse interval responses were measured.

Subjects. Volunteers comprised six healthy adults (five men and one woman) whose average age was $25 \pm 3 \mathrm{yr}$ $( \pm$ SEM). Subjects were studied supine, in a quiet darkened room after written, informed consent was obtained.

Measurements. A direct writing recorder was used to transcribe the electrocardiogram (lead II), beat-by-beat heart rate (cardiotachometer), neck chamber pressure, respiratory flow (Fleisch pneumotachograph, Instrumentation Associates, New York) and volume (electronic integration of respiratory flow), and expiratory $\mathrm{CO}_{2}$ concentration (infrared $\mathrm{CO}_{2}$ analyzer).

Control of respiration. Subjects wore nose clips and breathed through an airtight mouthpiece. Each subject's normal tidal volume was determined during quiet respiration, after the subject had become acclimatized to the apparatus and experimental environment. Inspiratory volume was displayed for the volunteer upon a calibrated oscilloscope; each subject was instructed to inspire to 100 , or to $150 \%$ of 
normal tidal volume during periods of data collection, at a comfortable respiratory rate.

Baroreceptor stimulation. Carotid baroreceptors were stimulated by applying suction to a neck chamber fashioned from an elliptical piece of sheet lead rimmed with sponge rubber (8). Neck suction was begun when rotation of a solenoid valve established continuity between the neck chamber and a continuous vacuum source. Stimuli were initiated electronically by tidal volume threshold crossings whose levels were established at the beginning of each experiment. The volunteer breathed normally between interventions and was not able to anticipate the onset of neck suction.

Stimuli of -30 or $-60 \mathrm{~mm} \mathrm{Hg}$ for $0.6 \mathrm{~s}$ were applied about 15 times each in early inspiration (after inspiration of about $5-10 \%$ of the tidal volume) or in expiration (after expiration of about $90-95 \%$ of the tidal volume). This experimental sequence also was repeated with the vacuum source turned off, to measure spontaneous fluctuations of pulse interval provoked by breathing. The subject was instructed to stop breathing at the conclusion of each respiratory cycle during which measurements were made. This did not alter the cadence of respiration, however, because the natural duration of the expiratory phase (defined as the interval between the end of one inspiration until the onset of the next [10]) was long, relative to the period of data collection (Table I).

Data analysis. Pulse interval (or P-P interval), ${ }^{1}$ was calculated from electrocardiogram R-wave threshold crossings by an on-line digital computer. The time from the onset of neck suction (or tidal volume threshold crossing without neck suction) until each successive $P$ wave, was calculated by subtracting the interval from the onset of the $P$ wave until the $R$ wave. In the subjects studied, neck suction did not provoke significant P-R interval prolongation. Serial P-P interval changes were plotted as a function of time from the inspiratory or expiratory threshold crossing until the $\mathrm{P}$ wave concluding each successive cycle. A composite curve was formed by averaging changes of pulse interval at 0.5-s intervals for $3 \mathrm{~s}$ after tidal volume threshold crossings. Statistical significance was determined with the analysis of variance with orthogonal contrasts, and paired, or unpaired $t$ tests (11). Differences were considered significant when $P$ was less than 0.05 .

\section{RESULTS}

Fig. 1 shows spontaneous changes of pulse interval occurring in one subject during a respiratory cycle and perturbations of this pattern provoked by neck suction applied in inspiration or expiration. Inspiration was associated with pulse interval shortening (cardioacceleration). Baroreceptor stimulation during inspiration (panel A) reduced inspiratory pulse interval shortening; baroreceptor stimulation during expiration (panel B) was associated with marked pulse interval prolongation. Average responses of all subjects are listed in Table $\mathrm{I}$ and are depicted in Figs. 2-5.

Baroreflex responses during inspiration. Fig. 2 shows spontaneous changes of pulse interval provoked by inspiration $(0 \mathrm{~mm} \mathrm{Hg})$, changes of pulse interval

${ }^{1}$ Abbreviation used in this paper: P-P interval, pulse interval.

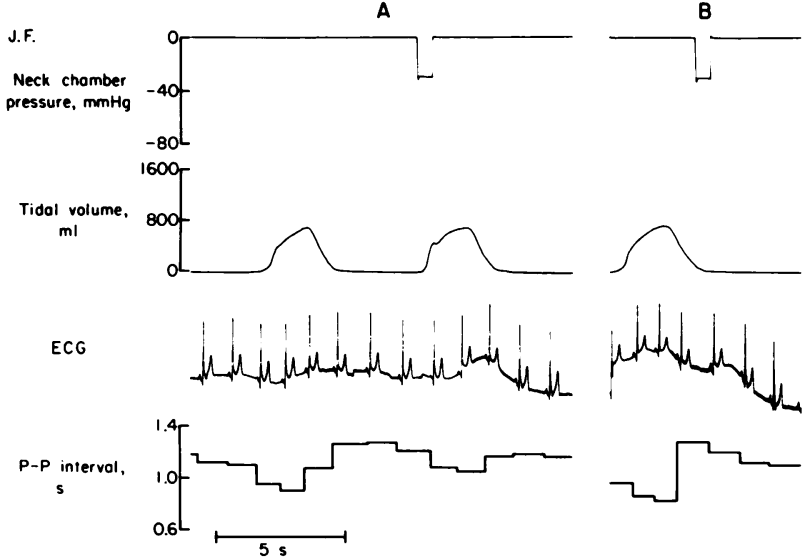

FigURE 1 Responses of one subject to baroreceptor stimulation begun in early inspiration (A) or expiration (B). Beatby-beat pulse intervals were drawn after the recording was made. Pulse interval prolongation associated with neck suction begun during inspiration was much less than that associated with neck suction begun during expiration.

after neck suction applied in early inspiration, and net sinus node responses to baroreceptor stimulation (difference). The net sinus node response was calculated by subtracting spontaneous pulse interval changes from those occurring after neck suction. Inspiration was associated with pulse interval shortening which persisted for about $3 \mathrm{~s}$. Neck suction $(30 \mathrm{~mm}$ $\mathrm{Hg}$ ) provoked brief, minor pulse interval prolongation and thus, transiently opposed, and reversed the normal pulse interval response to inspiration. The net sinus node response was pulse interval prolongation; this peaked at $1.25 \mathrm{~s}$ after the onset of neck suction and persisted for about $2.5 \mathrm{~s}$.

Baroreflex responses during expiration. Fig. 3 shows that spontaneous pulse interval shortening also occurred during expiration. Spontaneous changes of pulse interval were much smaller during expiration than inspiration and tended to be linear rather than parabolic. Absolute prolongation of the pulse interval by neck suction was much more striking when the stimulus was delivered during expiration than during inspiration (Fig. 2). The average control pulse interval (immediately before neck suction) was $1.04 \pm 0.07$ during inspiration, and $0.97 \pm 0.04 \mathrm{~s}$ during expiration $(P>0.1$, paired $t$ test). Only the net sinus node responses to neck suction are depicted in subsequent figures.

Responses to different intensities of baroreceptor stimulation. Fig. 4 shows net sinus node responses to -30 or $-60 \mathrm{~mm} \mathrm{Hg}$ delivered during inspiration or expiration. Responses to $-30 \mathrm{~mm} \mathrm{Hg}$ (upper panel) were significantly $(P<0.001)$ less when stimuli were delivered in early inspiration than in expiration. Sinus node responses to $-60 \mathrm{~mm} \mathrm{Hg}$ (lower panel) 
TABLE I

Average Responses of All Subjects

\begin{tabular}{|c|c|c|c|c|c|c|}
\hline & \multicolumn{6}{|c|}{ Stimulus* to P-wave interval, $s$} \\
\hline & 0.25 & 0.75 & 1.25 & 1.75 & 2.25 & 2.75 \\
\hline \multicolumn{7}{|l|}{$\begin{array}{l}100 \% \text { Tidal volume } \\
0 \mathrm{~mm} \mathrm{Hg}\end{array}$} \\
\hline A Inspiration & $-57 \pm 13$ & $-55 \pm 20$ & $-100 \pm 24$ & $-124 \pm 38$ & $-101 \pm 29$ & $-16 \pm 52$ \\
\hline B Expiration & $160 \pm 56$ & $146 \pm 33$ & $169 \pm 29$ & $154 \pm 43$ & $100 \pm 26$ & $119 \pm 45$ \\
\hline \multicolumn{7}{|l|}{$-30 \mathrm{~mm} \mathrm{Hg}$} \\
\hline C Inspiration & $-42 \pm 11$ & $0 \pm 29$ & $44 \pm 52$ & $-57 \pm 25$ & $-60 \pm 28$ & $-63 \pm 22$ \\
\hline D Expiration & $165 \pm 66$ & $288 \pm 96$ & $375 \pm 58$ & $215 \pm 55$ & $163 \pm 26$ & $141 \pm 49$ \\
\hline \multicolumn{7}{|l|}{$-60 \mathrm{~mm} \mathrm{Hg}$} \\
\hline E Inspiration & $-25 \pm 25$ & $38 \pm 21$ & $164 \pm 57$ & $67 \pm 54$ & $-46 \pm 28$ & $-23 \pm 51$ \\
\hline F Expiration & $185 \pm 74$ & $314 \pm 77$ & $433 \pm 62$ & $321 \pm 45$ & $142 \pm 47$ & $171 \pm 19$ \\
\hline \multirow{2}{*}{\multicolumn{7}{|c|}{$\begin{array}{l}150 \% \text { Tidal volume } \\
0 \mathrm{~mm} \mathrm{Hg}\end{array}$}} \\
\hline & & & & & & \\
\hline G Inspiration & $-67 \pm 16$ & $-90 \pm 30$ & $-141 \pm 37$ & $-112 \pm 62$ & $-148 \pm 50$ & $-95 \pm 50$ \\
\hline H Expiration & $179 \pm 94$ & $113 \pm 73$ & $160 \pm 80$ & $117 \pm 75$ & $62 \pm 72$ & $133 \pm 71$ \\
\hline \multicolumn{7}{|l|}{$-30 \mathrm{~mm} \mathrm{Hg}$} \\
\hline I Inspiration & $-55 \pm 10$ & $18 \pm 33$ & $3 \pm 54$ & $-92 \pm 44$ & $-119 \pm 36$ & $-128 \pm 30$ \\
\hline J Expiration & $157 \pm 95$ & $111 \pm 35$ & $238 \pm 69$ & $29 \pm 77$ & $-12 \pm 71$ & $86 \pm 60$ \\
\hline
\end{tabular}

Average pulse interval responses ( $\pm 1 \mathrm{SEM}$ ), in $\mathrm{ms}$, for all subjects, to all interventions. Net sinus node responses (not listed) were obtained by subtracting spontaneous changes of pulse interval $(0 \mathrm{~mm} \mathrm{Hg}$ ) from those occurring after neck suction. $P$ values (analysis of variance with orthogonal contrasts): A vs. $\mathrm{G}=\mathrm{NS}$; $B$ vs. $\mathrm{H}=\mathrm{NS}$; $\mathrm{C}$ vs. $\mathrm{D}=<0.001$; $C$ vs. $E=<0.005$; $C$ vs. $\mathrm{I}=\mathrm{NS}$; $\mathrm{D}$ vs. $\mathrm{F}=\mathrm{NS}$; $\mathrm{D}$ vs. $\mathrm{J}=<0.001 ; \mathrm{E}$ vs. $\mathrm{F}=<0.001$.

* Spontaneous pulse interval changes with inspiration or expiration are denoted by " $0 \mathrm{~mm} \mathrm{Hg}$ ". The timing of these measurements was exactly the same as that after neck suction; there was, however, no superimposed baroreflex stimulus.

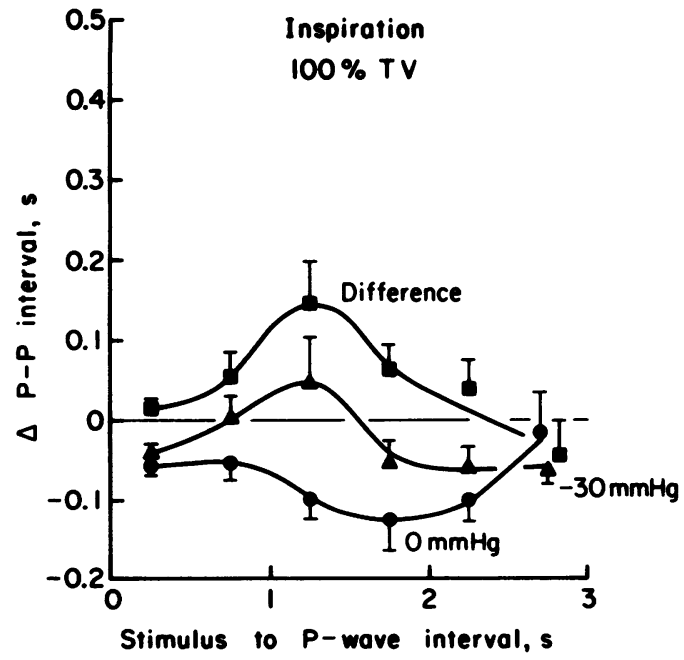

Figure 2 Average P-P interval changes of all subjects during inspiration. Spontaneous changes provoked by inspiration $(0 \mathrm{~mm} \mathrm{Hg})$ are shown as circles. Changes of P-P interval after neck suction $(30 \mathrm{~mm} \mathrm{Hg}$ ) are shown as triangles. The net sinus node response to baroreceptor stimulation (difference) was calculated by subtracting responses without neck suction from responses with neck suction. Brackets encompass 1 SEM. TV, tidal volume. were greater than those to $-30 \mathrm{~mm} \mathrm{Hg}$, in both phases of respiration. However, in contrast with responses to -30 , responses to $-60 \mathrm{~mm} \mathrm{Hg}$ were not significantly less during inspiration. This suggests that inspiratory inhibition of baroreflex responses can be overridden by intense baroreceptor stimulation.

Baroreflex responses at different tidal volumes. The influence of tidal volume upon ventilation is indicated in Table II. Supranormal (150\%) tidal volume was associated with a slightly slower average respiratory rate and more prolonged inspiration; there was no significant reduction of end-tidal $\mathrm{CO}_{2}$ concentration, or change of functional residual capacity. Average control pulse intervals before stimuli delivered during late expiration were comparable with 100 and $150 \%$ tidal volumes $(1.06 \pm 0.07$ vs. $1.10 \pm 0.07 \mathrm{~s}, P>0.5)$. Also, spontaneous pulse interval changes following expiration were not influenced significantly by the depth of respiration (Table I).

Fig. 5 shows average sinus node responses to -30 $\mathrm{mm} \mathrm{Hg}$ delivered during respirations of 100 or $150 \%$ of each subject's normal tidal volume. Responses to stimuli begun during early inspiration (upper panel) were not influenced by the depth of the ensuing 
breath. Responses to stimuli delivered during expiration (lower panel), however, were significantly ( $P$ $<0.001$ ) less after a tidal volume of 150 than $100 \%$ of normal.

\section{DISCUSSION}

We have studied the quantitative relationship that exists between respiratory and baroreceptor influences acting upon the sinus node, in six normal human volunteers. The principal findings are that inspiration reduces sinus node responses to moderate baroreflex stimuli, but not to intense stimuli, and that respiratory baroreflex suppression is proportional to the depth of inspiration.

In the subjects studied, baroreceptor stimulation altered the patterns of sinus node responses to respiratory activity (Figs. 2 and 3). During quiet breathing, inspiration was associated with pulse interval shortening. Moderate baroreceptor stimulation opposed and transiently reversed inspiratory pulse interval shortening, and intense baroreceptor stimulation caused major pulse interval prolongation. Spontaneous pulse interval shortening occurred also after expiration. Moderate and intense expiratory baroreflex stimuli provoked pulse interval prolongation, which was significantly $(P<0.001$, Table I) greater than that occurring after inspiratory baroreceptor stimulation.

We considered the possibility that the apparent inhibition of baroreflex responses during inspiration might be due merely to algebraic summation of opposing sinus node responses to inspiration and baroreceptor stimulation. This did not appear likely, because when spontaneous pulse interval changes caused by respiration were subtracted from responses

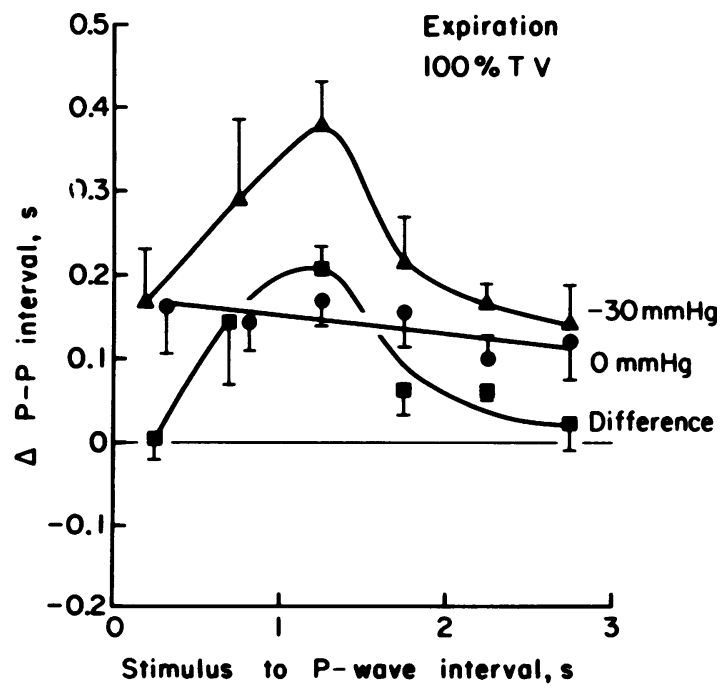

FIGURE 3 Average P-P interval changes during expiration. TV, tidal volume.

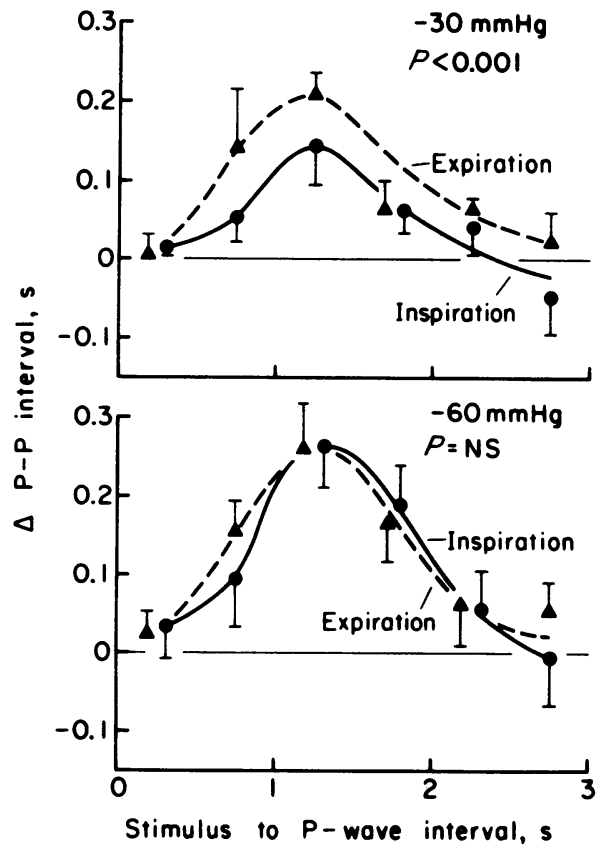

Figure 4 Average net $\mathrm{P}-\mathrm{P}$ interval responses to two intensities of neck suction.

following moderate baroreceptor stimulation, the net sinus node response was still significantly $(P<0.001$ Fig. 4, upper panel) less when stimuli were begun in inspiration than expiration.

Respiratory activity did not alter sinus node responses to intense $(-60 \mathrm{~mm} \mathrm{Hg})$ baroreceptor stimulation, however (Fig. 4, lower panel). An earlier study (12) showed that pulse interval prolongation caused by neck suction of 30 and $60 \mathrm{~mm} \mathrm{Hg}$ is nearly linearly related to stimulus intensity. This provides evidence in favor of a true central autonomic interaction; if inspiratory suppression were due merely to a constant level of opposition, the same degree of inhibition should have been observed at all levels of

TABLE II

Influence of Tidal Volume upon Ventilation

\begin{tabular}{|c|c|c|c|}
\hline & \multicolumn{2}{|c|}{ Tidal volume } & \multirow[b]{2}{*}{$P$} \\
\hline & $100 \%$ & $150 \%$ & \\
\hline $\begin{array}{l}\text { Respiration frequency, } \\
\text { breaths/min }\end{array}$ & $10.80 \pm 1.0$ & $9.10 \pm 0.5$ & $<0.05$ \\
\hline Duration of inspiration, $s$ & $1.90 \pm 0.2$ & $2.30 \pm 0.3$ & NS \\
\hline Tidal volume, liters & $0.75 \pm 0.04$ & $1.15 \pm 0.06$ & $<0.001$ \\
\hline $\begin{array}{l}\text { End-tidal } \mathrm{CO}_{2} \\
\text { concentration, \% }\end{array}$ & $5.12 \pm 0.13$ & $4.99 \pm 0.15$ & NS \\
\hline
\end{tabular}

Average responses $( \pm 1 \mathrm{SEM})$ for all subjects. Statistical significance was determined with the paired $t$ test. 


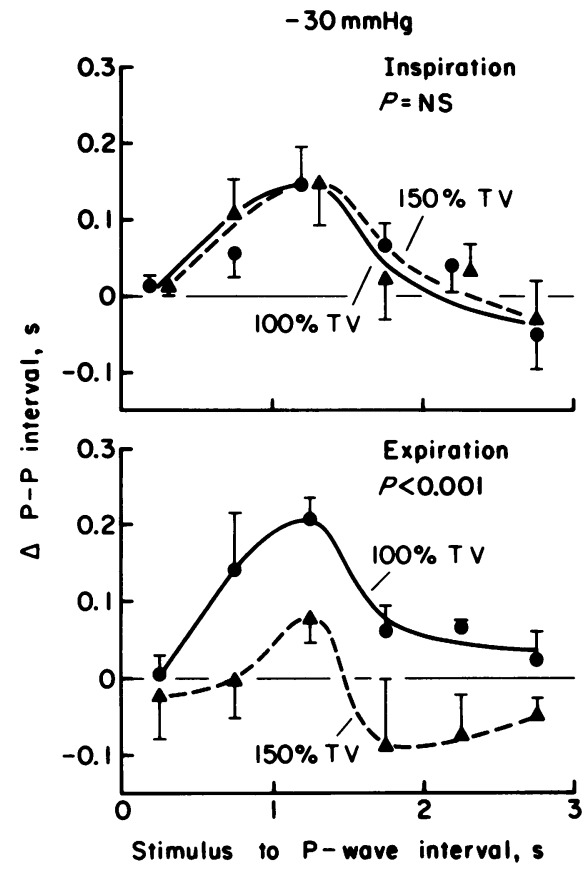

FIGURE 5 Average net baroreflex P-P interval responses associated with normal and supranormal tidal volumes (TV).

barosensory input. This was not observed; inspiratory baroreflex inhibition was overridden by intense baroreceptor stimulation. A related observation was made by Anrep et al. (13) who found that sinus arrhythmia disappears at very high arterial pressures in anesthetized dogs. This type of central interaction endows the respiratory-baroreflex control system with a flexibility which enables it to respond appropriately to different afferent carotid baroreceptor inputs. When arterial pressure is high, important baroreflex adjustments are not inhibited by inspiration.

Two tidal volumes, 100 and $150 \%$ of normal, were used to evaluate the importance of the depth of inspiration upon respiratory-baroreflex interactions. Inspiratory baroreflex responses were not influenced by tidal volume (Fig. 5, upper panel). The 0.6-s baroreflex stimulus used in this study occupied approximately the first one-third of inspiration (Fig. 1, Table II) and the resulting perturbation of pulse interval extended slightly beyond the end of inspiration. We suggest that the sinus node response to the baroreflex stimulus was determined before the increment of inspiration occurred and was not altered by the subject's intention to breathe deeply.

Expiratory baroreflex responses on the other hand, were significantly $(P<0.001)$ inhibited by supranormal inspiration; the onset of pulse interval prolongation was delayed, its extent was reduced, and it persisted for a shorter duration (Fig. 5, lower panel). Since volunteers breathed out to the same functional residual capacity, this suggests that the degree of respiratory baroreflex suppression is proportional to the level of inspiration. Spontaneous sinus node responses were comparable after normal and supranormal tidal volumes (Table I, B and $\mathrm{H}$ ) and therefore, suppression of expiratory baroreflex responses by supranormal inspiration points toward the existence of central baroreflex inhibition, in the absence of detectable alteration of normal sinus node neural regulation. Klevans and Gebber (14) have shown that electrical stimulation of certain forebrain structures in anesthetized cats may alter significantly sinus node responses to baroreceptor stimulation without provoking significant changes of heart rate or arterial pressure. A related phenomenon may explain the present results obtained from unsedated human volunteers.

Inhibition of baroreflex responses persisted for at least several seconds into expiration. Since afferent activity from pulmonary stretch receptors $(15,16)$ and efferent activity from respiratory centers (17) occur almost exclusively during inspiration, it is ur.likely that these influences explain fully inhibition of baroreflex responses persisting into the middle of the expiratory phase. A possibly related phenomenon was described by Gimpl and associates (18) who documented persistence of central baroreflex (electrical stimulation of the aortic nerve) inhibition after removal of the opposing stimulus (electrical stimulation of the posterior hypothalamus). However, we cannot exclude the possibility that persistence of baroreflex inhibition into mid-expiration resulted from expiratory activation of deflation receptors $(15,19,20)$.

The present study was not designed to explore all possible explanations for cardioacceleration occurring during inspiration. Studies from this laboratory (21) suggest that in man, baroreflex inhibition due to stimulation of cardiopulmonary receptors does not contribute. Pulse interval prolongation caused by intravenous phenylephrine injections was not modified by moderate (1-9 $\mathrm{mm} \mathrm{Hg}$ ) changes of central venous pressure caused by lower body suction or leg elevation.

Our study does not define the central mechanism responsible for respiratory baroreflex modulation. We have not excluded the possibility that baroreflex suppression during inspiration results from an automatic rhythm common to respiratory and vagal centers (22). Since no attempt was made to control respiratory frequency, vagal nuclei may have been influenced by impulses arising in the respiratory center as well as from chest and lung expansion.

It is unlikely that diminished baroreflex responses were secondary to small fluctuations of arterial pressure associated with breathing, because both intensities of baroreceptor stimuli used are above the carotid baro- 
receptor activation threshold for man, and the lower intensity, $-30 \mathrm{~mm} \mathrm{Hg}$, is on the linear portion of the sinus node response relation to neck suction (12).

Earlier studies from this, and other laboratories support the validity of using pulse interval as an index of efferent cholinergic activity. Katona et al. (3) demonstrated in chloralose-anesthetized dogs, that pulse interval during respiration or baroreceptor stimulation can be predicted closely from efferent vagal nerve activity, by using a linear equation. We have shown that changes of pulse interval provoked by brief neck suction in healthy young adults appear to be due entirely to fluctuations of efferent cholinergic activity $(8,23)$.

Our study relates to central interactions involving respiration and carotid baroreceptor inputs. Similar techniques could be used to elucidate other reflex interactions in man. We have looked at responses of only one effector, the sinus node. Daly et al. (24) have demonstrated in anesthetized dogs that respiration influences arterial baroreflex control of systemic vascular resistance; this possible interrelation is not known to have been studied in man.

\section{ACKNOWLEDGMENTS}

The authors acknowledge their appreciation to Dr. Francois M. Abboud for his encouragement and critical review of this manuscript, and to Mr. Michael S. Cavanaugh and Mr. Sukgi Choi for their skilled technical assistance.

This research was supported by grants from the American Lung Association of Iowa, Veterans Administration, and the National Institutes of Health, (HL 14388 and HL 18083).

\section{REFERENCES}

1. Rijlant, P. 1936. L'arythmie cardiaque respiratoire. Seances Soc. Biol. Fil. 123: 997-1001.

2. Iriuchijima, J., and M. Kumada. 1964. Activity of single vagal fibers efferent to the heart. Jpn. J. Physiol. 14: 479487.

3. Katona, P. G., J. W. Poitras, G. O. Barnett, and B. S. Terry. 1970. Cardiac vagal efferent activity and heart period in the carotid sinus reflex. Am. J. Physiol. 218: 1030-1037.

4. Koepchen, H. P., H. D. Lux, and P-H. Wagner. 1961. Untersuchungen über Zeitbedarf und zentrale Verarbeitung des pressoreceptorischen Herzreflexes. Pflüegers Archiv. Gesamte Physiol. Menschen Tiere. 273: 413-430.

5. Haymet, B. T., and D. I. McCloskey. 1975. Baroreceptor and chemoreceptor influences on heart rate during the respiratory cycle in the dog. J. Physiol. (Lond.). 245: 699-712.

6. Davidson, N. S., S. Goldner, and D. I. McCloskey. 1976. Respiratory modulation of baroreceptor and chemoreceptor reflexes affecting heart rate and cardiac vagal efferent nerve activity. J. Physiol. (Lond.). 259: 523-530.

7. Smyth, H. S., P. Sleight, and G. W. Pickering. 1969.
Reflex regulation of arterial pressure during sleep in man. A quantitative method of assessing baroreflex sensitivity. Circ. Res. 24: 109-121.

8. Eckberg, D. L., M. S. Cavanaugh, A. L. Mark, and F. M. Abboud. 1975. A simplified neck suction device for activation of carotid baroreceptors. J. Lab. Clin. Med. 85: 167-173.

9. Eckberg, D. L. 1976. Temporal response patterns of the human sinus node to brief carotid baroreceptor stimuli. J. Physiol. (Lond.). 258: 769-782.

10. Cohen, M. I. 1968. Discharge patterns of brain-stem respiratory neurons in relation to carbon dioxide tension. J. Neurophysiol. 31: 142-165.

11. Huntsberger, D. V., and P. E. Leaverton. 1970. Statistical inference in the biomedical sciences. Allyn \& Bacon, Inc., Boston. 269 pp.

12. Eckberg, D. L. 1977. Baroreflex inhibition of the human sinus node: Importance of stimulus intensity, duration, and rate of pressure change. J. Physiol. (Lond.). In press.

13. Anrep, G. V., W. Pascual, and R. Rössler. 1936. Respiratory variations of the heart rate. I-The reflex mechanism of the respiratory arrhythmia. Proc. R. Soc. Lond. B. Biol. Sci. 119: 191-217.

14. Klevans, L. R., and G. L. Gebber. 1970. Facilitatory forebrain influence on cardiac component of baroreceptor reflexes. Am. J. Physiol. 219: 1235-1241.

15. Adrian, E. D. 1933. Afferent impulses in the vagus and their effect on respiration. J. Physiol. (Lond.). 79: $332-358$.

16. Langrehr, D. 1964. Receptor-Afferenzen im Halsvagus des Menschen. Klin. Wochenschr. 42: 239-244.

17. Adrian, E. D., and D. W. Bronk. 1928. The discharge of impulses in motor nerve fibres. Part I. Impulses in single fibres of the phrenic nerve.J. Physiol.(Lond.). 66: 81-101.

18. Gimpl, M. P., A. L. Brickman, M. P. Kaufman, and N. Schneiderman. 1976. Temporal relationships of barosensory attenuation in conscious rabbits. Am.J. Physiol. 230: 1480-1486.

19. Paintal, A. S. 1955. Impulses in vagal afferent fibres from specific pulmonary deflation receptors. The response of these receptors to phenyl diguanide, potato starch, 5-hydroxytryptamine and nicotine, and their role in respiratory and cardiovascular reflexes. Q. J. Exp. Physiol. Cogn. Med. Sci. 40: 89-111.

20. Paintal, A. S. 1957. The location and excitation of pulmonary deflation receptors by chemical substances. Q. J. Exp. Physiol. Cogn. Med. Sci. 42: 56-71.

21. Takeshita, A., A. L. Mark, D. L. Eckberg, and F. M. Abboud. 1977. Effects of cardiopulmonary baroreceptor activity on arterial baroreflex sensitivity in humans. Fed. Proc. 36: 487.

22. Traube, L. 1865. Ueber periodische Thätigkeits-Aeusserungen des vasomotorischen und Hemmungs-Nervencentrums. Centbl. Med. Wiss. 56: 881-885.

23. Eckberg, D. L., F. M. Abboud, and A. L. Mark. 1976. Modulation of carotid baroreflex responsiveness in man: effects of posture and propranolol. J. Appl. Physiol. 41: 383-387.

24. Daly, M. deB., J. L. Hazzledine, and A. Ungar. 1967. The reflex effects of alterations in lung volume on systemic vascular resistance in the dog. J. Physiol. (Lond.). 188: $331-351$. 